\title{
La desaparición - o tal vez no - de la educación de personas adultas. El caso de Andalucía
}

The missing - or maybe not - of adult education. The case of Andalusia

Emilio Lucio-Villegas*

Universidad de Sevilla

Resumo Este artículo trata de considerar las diferencias entre los conceptos de Aprendizaje a lo largo de la vida y Educación de personas adultas, prestando especial atención a la evolución de la educación de personas adultas en Andalucía (Sur de España). Desde 1982, Andalucía tiene completa autonomía para legislar en educación. Desde esa fecha se desarrolló un proyecto para superar las altas tasas de analfabetismo, desarrollando un plan específico en el campo de la educación de personas adultas íntimamente ligado con los ayuntamientos y las comunidades: el llamado modelo territorial. A partir de 1990 hay un cambio radical en las políticas en educación de personas adultas. Los años 90 representan un cambio que marca un nuevo modelo de educación de personas adultas caracterizado por tomar distancia de las comunidades y centrarse más en el curriculum escolar.

PALAVRAS-CHAVE: Educación de personas adultas; Aprendizaje a lo largo de la vida; Modelo territorial; Alfabetización.

Abstract This paper tries to consider differences between the concepts and practices of the Lifelong Learning and Adult Education paying attention on the evolution of Education of the adults in Andalusia (south of Spain). From 1982 on, Andalusia has full autonomy for legislating in education. From this date it started the project to overcome the high rates of illiteracy developing a specific project in the field of adult education closely related to municipalities and communities in a model called: the territorial model. From 1990 onwards there was a radical change in the policy on adult education. The 90 ' represented a change and marked a specific model of adult education, characterised by its distance from communities and more centred on curricula.

KEYWORDS: Adult education; Lifelong learning; Territorial model; Literacy. 


\title{
Introducción
}

El principal objetivo de este artículo es presentar la evolución de la educación de personas adultas (EA en adelante) ejemplificando el caso de Andalucía (sur de España) desde 1982. La idea principal es que un marco teórico específico - conceptos como Aprendizaje a lo largo de la vida (ALV en adelante) o EA - están relacionados con determinados momentos históricos. Tuvimos una época en la que el concepto de EA parecía ser dominante y ahora se encuentra oscurecido por el concepto de ALV con su carga ideológica de búsqueda de rentabilidad económica de cualquier actuación educativa. Para dar una explicación de ese carácter contextual de los grandes conceptos - educativos o de otro tipo - podemos remitirnos al final de la II Guerra Mundial, cuando en un momento de búsqueda de las raíces del mal y de construcción de un mundo más justo - como una nueva Ilustración - se definió el concepto de Educación Permanente que Lengrand (1973) definió indicando:

\begin{abstract}
que la única cosa provechosa que un educador puede hacer por otro hombre, y concretamente por un adulto, consiste en facilitarle los instrumen $\neg$ tos y colocarle en situaciones en las que pueda, a partir de los elementos de su propia condición, a partir de lo cotidiano de sus experiencias, de sus luchas, de sus éxitos y de sus fracasos, construirse un saber propio y una reflexión personal, y con un esfuerzo progresivo tomar posesión de los elementos de su personalidad, ocupando sus dimensiones y dándoles una forma y una expresión (p. 20).
\end{abstract}

En la actualidad vivimos en la era del ALV. Este concepto está marcando los desarrollos en todo el sistema educativo - formal, no formal o informal - desde una perspectiva muy diferente de aquella a la que se refería Lengrand más arriba.

En definitiva, en este artículo, intentaré explorar las diferencias entre los tradicionales conceptos de EA como elementos de liberación y construcción de la creatividad personal y social y aquellos ligados al ALV desde su perspectiva de que la educación - de cualquier tipo - debe tener un carácter aplicado e instrumental que persigue otros fines diferentes a los propiamente educativos. Tras eso, presentaré brevemente la evolución de los procesos educativos para personas adultas en Andalucía. Por último, trataré de relacionar esta evolución con la transición de la EA y al ALV.

\section{Qué denomino educación de personas adultas}

Definir la EA puede ser muy complicado en ocasiones. En otro lugar ( $\mathrm{Lu}-$ cio-Villegas, 2015) lo he considerado un concepto muy difuso que está relacionado con diferentes significados y prácticas. De alguna forma, podemos definirla siguiendo a McCullough (en Jarvis, 1989).

Extraer la educa $\neg$ ción de adultos del mundo social que la rodea - o al menos diferenciar la educación de adultos de su medio social es tan difícil como determinar cuantos ángeles pueden bailar en la cabeza de un alfiler. ¿La educación de adultos es una práctica o un programa? ¿Una metodología o una organización? ¿Una ciencia o un sistema? ¿Un proceso o una profesión? ¿La educación de adultos 
es diferente de la educación contiᄀnua, la educación vocacional, la educación superior?... ¿Existe siquiera la educación de adultos? (p. 23).

En un amplio rango de actividades y teorías me gustaría destacar algunos aspectos que puede explicar que es lo que considero EA. Cuando hablo de EA, quiero referirme a un tipo de educación dirigida a jóvenes y personas adultas - en una sociedad que culturalmente ha definido lo que entiende por joven o por persona adulta. Para mí, se encuentra ligada a lo que se entiende como Educación Popular, que puede ser caracterizada como: i) basada en los intereses reales de las personas; ii) comprometida con el cambio social; iii) con un enfoque eminentemente colectivo; y iv) conectando la educación y la acción social (Crowther, Johnston, Martin \& Merrill, 2006).

Por otro lado, he definido la EA muy relacionada con la vida cotidiana en contraposición al ALV cuyos concepto y prácticas

\begin{abstract}
parecen haber olvidado que la vida es algo más que el mundo del trabajo. La vida incluye: familiares, relaciones con los compañeros y amigos; padres e hijos; vacaciones y trabajo; deseos e ilusiones; felicidad y tristeza; buenas y malas decisiones; emociones y sentimientos. [...] Los conceptos y prácticas del ALV parecen haber olvidado que la vida completa de las mujeres y los hombres es la sustancia de la que la que está hecha la educación de personas adultas. (LucioVillegas, 2009, p. XIV).
\end{abstract}

También es posible señalar la EA desde la perspectiva de una metodología específica que liga la vida real y cotidiana de las personas con el curriculum y los materiales utilizados. En una perspectiva freireana puede decirse que se trata de leer y decir el mundo al mismo tiempo que las personas leen y dicen palabras y frases. Como señala Freire (1985) las personas son más conscientes de su situación cuando se parte de las palabras generadoras como la base de la EA.

Hill (2010) plantea una serie de cuestiones muy interesantes a la hora de elaborar/establecer una definición:

¿Quién está aprendiendo qué? ¿De quién? ¿Con qué metodología? ¿Para qué propósito? ¿Bajo qué circunstancias? ¿Y con qué consecuencias? [...] se pueden ampliar estos interrogantes preguntando, también, quienes pagan (p. 185).

Las respuestas que demos a las cuestiones que plantea este autor pueden ayudarnos a considerar la EA, desde una cierta perspectiva.

Finalmente, creo que la EA se organiza también en el encuentro entre la cultura popular y la cultura clásica en el sentido que propuso Raymond Williams cuando habló sobre el concepto de criticismo,

Criticismo como una definición de respuesta consciente $[\ldots]$ Incluyendo a menudo, necesariamente, respuestas positivas o negativas, una práctica definida, unas activas y complejas relaciones con su situación y contexto. (1989, p. 86). 


\section{Qué denomino aprendizaje a lo largo de la vida}

La introducción del concepto de aprendizaje para situarlo en el centro de los procesos educativos pareció un asunto muy positivo. Pero lo primero que las políticas y prácticas del ALV hicieron fue transferir la responsabilidad de ese aprendizaje a las personas concebidas de forma individual "quienes, en última instancia, son responsables de perseguir su propio aprendizaje” (CE, 2000, p. 5). De alguna forma, esto implica que un derecho - el derecho a la educación como derecho universal de todas las personas - se convierte en una mercancía y, como han señalado Gomes y LucioVillegas (2009) "promover la expansión de las oportunidades de educación y aprendizaje [...] no ha garantizado igual acceso para todas las personas" (p. 75).

Otro asunto importante es que las políticas y prácticas del ALV parecen haberse relacionado sólo con el mundo del trabajo en un sentido muy estrecho - nada que ver, por ejemplo, con lo que señalaba Gelpi (cf.1990, 2004, 2008). De hecho la noción de ALV comúnmente usada en el contexto europeo deriva de la Estrategia Europea para el Empleo que señalaba que sólo la educación y el aprendizaje ayudaban a mantener la competitividad económica y la empleabilidad (CE, 2000). Pero, además, la empleabilidad no es un empleo, es la posibilidad de tenerlo.

Si continuamos con la definición de los documentos oficiales, el ALV trata de alcanzar dos grandes objetivos centrados en promover la ciudadanía activa y promover la empleabilidad (CE, 2000). Como ya hemos desarrollado en otro lugar ( $\mathrm{Lu}^{-}$ cio-Villegas, 2014) el segundo objetivo se convirtió en dominante, dejando el primero en formulaciones muy vagas referentes a la cohesión social - o al sueño de que exista una sociedad sin conflictos de clase en las formaciones sociales existentes en la actualidad. Las consecuencias del abandono de las políticas educativas, y no sólo, referidas a la ciudadanía activa está teniendo consecuencias en el panorama actual caracterizado por el incremento del fascismo y la condena de cualquier forma de diversidad o divergencia con el pensamiento dominante - cada vez más estrecho, por cierto.

Me gustaría hacer dos comentarios más sobre las políticas y prácticas del ALV que marcan nuestro presente y nuestro futuro educativo, al menos por ahora. En primer lugar sobre los fines que la Unión Europea intenta alcanzar con estas políticas: i) reducir la escasez de trabajadores elevando el nivel de destrezas en la fuerza de trabajo, ii) encarar el problema del alto porcentaje de abandono escolar ofreciendo una segunda oportunidad para recibir alguna cualificación, iii) reducir los niveles de pobreza y exclusión social, iv) "incrementar la integración de los inmigrantes en la sociedad y en el mercado de trabajo" (CE, 2007, p. 3), v) incrementar la participación en procesos de ALV. Considero que, a partir de estos objetivos, el proyecto educativo nacido de la idea de Ilustración ha desaparecido. El asunto más importante parece ser, en este momento, acumular, en una lógica economicista, diplomas y habilidades que permitan a las personas ser empleables, estar preparadas para formar parte del mercado de trabajo como ejercito industrial de reserva.

El segundo asunto tiene que ver con los llamados seis mensajes clave que resumo: i) nuevas herramientas básicas para todos, ii) mayor inversión en recursos humanos, iii) innovación en la enseñanza y el aprendizaje, iv) valoración del aprendizaje, 
v) repensar la guía y el consejo, y vi) acercar el aprendizaje al hogar. Lo primero que quiero destacar es que cuando se habla de nuevas herramientas en este contexto, se está hablando, fundamentalmente, de las Tecnologías de la Información y la Comunicación. La idea de acercar el aprendizaje al hogar supone potenciar el aprendizaje informal, pero el peligro es que esta nueva valoración del aprendizaje informal parece estar hecha en conflicto con el sistema escolar. Finalmente, en relación a la guía y los procesos de consejo, puede ser interesante reflexionar desde un punto de vista foucaultiano y las relaciones entre los procesos de guía y el poder (cf., Nicoll y Fejes, 2008: Edwards, 2008).

Lima ha denominado a este tipo de educación como educación contable muy cercana a la noción de educación bancaria definida por Freire (e.g. 1985). Para Lima (2000):

En educación, los discursos gerenciales han ocupado la posición que previamente era asumida por las teorías educativas y el pensamiento pedagógico, construyendo narrativas de tipo gerencial que legitiman un nuevo orden basado en el mercado y en los sectores productivos privados, en la competencia económica y en la organización centrada en el cliente (p. 243).

\section{Una breve introducción sobre España}

El analfabetismo ha sido un importante asunto durante la historia de España, y un elemento significativo a la hora de analizar la situación de la EA en el devenir histórico-educativo del Estado. En los comienzos del siglo XX el porcentaje de personas analfabetas rondaba el 60\% - que es mucho más alto, por ejemplo, que los índices que presentaba Suecia en el siglo XVIII (Viñao, 1990). Sólo durante el tiempo de los gobiernos de la II República - sobre todo a partir de la victoria del Frente Popular en febrero de 1936 - se realizó un gran esfuerzo para reducir los índices de pobreza y analfabetismo. Con la excepción de este período - hasta la democracia, como veremos un poco más adelante - la educación en general y aquella pensada para personas adultas, nunca fue un asunto de preocupación para los políticos, ni un asunto esencial en el conjunto de políticas del Estado - esto último sigue siendo válido a fecha de hoy. En los años que precedieron a la Segunda República, los terratenientes - no existía una clase de industriales poderosa - no estaban interesados en la educación de las personas. Más tarde, en los negros años de la Dictadura la represión de la cultura, la educación, los sindicatos o cualquier otra forma de organización popular, era un asunto común.

La educación de personas adultas vuelve a convertirse en un asunto de interés coincidiendo con la restauración de la democracia - sobre todo durante el período conocido como La Transición, 1975-1978. Durante esos años y los primeros del Estado constitucional, después de 1978, la educación se convirtió en una importante preocupación, aun cuando existían importantes preocupaciones: hasta 1981 existió un cierto riesgo de involución política, más tarde, cuando España de unió a la Unión Europea - 1986 - se estaba luchando contra muy serios problemas económicos. De alguna forma, el tradicional atraso educativo - sobre todo en cuanto a medidas legislativas, pero no en cuanto a la creatividad social - continuó durante ese período. De 
hecho la primera ley educativa de la democracia es de 1990 - Ley Orgánica de Ordenación General del Sistema Educativo (LOGSE), es aprobada 20 años después de la Ley General de Educación de 1970 y 15 años después de iniciado el llamado proceso de Transición.

En este escenario donde la primacía del desarrollo era la creatividad social, es muy fácil entender como la EA se convirtió en educación popular. Creció sin un estricto control del Estado - que sólo llegaría en 1990 con la LOGSE. De hecho, podemos afirmar que el desarrollo creativo de la EA durante ese período se debe a su nacimiento a partir de los movimientos ciudadanos de todo tipo, y de la falta de control sobre estos procesos creativos.

En resumen, se puede considerar que no existe una larga tradición relacionada con la EA en España. Las más interesantes y creativas iniciativas tuvieron lugar cuando la EA no era controlada por la legislación y el poder. A partir de 1990, el asunto más importante pasó a ser la educación secundaria y el despachar títulos para homologarse al resto de países europeos. Desde el año 2000, el foco está en cumplir los preceptos de las políticas y prácticas del ALV. Estas políticas se están poniendo en práctica en los últimos 17 años en un contexto que sigue marcado por los altos niveles de analfabetismo y un preocupante - y vergonzoso para los poderes públicos - abandono escolar temprano.

\section{¿Por qué me centro en Andalucía?}

La batalla por la democracia en España tiene un elemento esencial que a veces es olvidado. Esa batalla, algunas veces sangrienta y siempre dolorosa, es indisociable de la lucha de las personas por recuperar la diversidad cultural, lingüística, social y nacional que la Dictadura había robado de todos los rincones del Estado. La construcción del Estado Constitucional desde 1978 culmina con la imparable explosión de la diversidad existente en cada esquina. De hecho, se puede decir que España es una nación de naciones que integra diversos territorios muy diversos, denominados - en la terminología legal - Comunidades Autónomas.

En esta dirección, procurar una uniformidad en el campo de la EA es bastante difícil. En España, asuntos como sanidad, transportes públicos, impuestos, educación, etc. se encuentran total o parcialmente en las manos de los gobiernos de las Comunidades Autónomas. En el caso de la educación, cada territorio tiene gran poder legislativo. Un ejemplo de ello puede ser la inexistencia de una ley de educación de personas adultas a nivel del Estado, y la existencia de diversas a nivel de territorios: Galicia (1992), Valencia (1995), Islas Canarias (2003) o Islas Baleares (2006).

En resumen, cada territorio tiene la posibilidad de implementar sus propias medidas legislativas. En el caso concreto de la EA y el ALV esta implementación y desarrollo suele ser realizada por los Departamentos de Educación, aunque también puede depender de Empleo, Bienestar Social y similares. Esto mismo hace que sea algo complicado presentar un territorio como ejemplo. En ese sentido es importante destacar que Andalucía, en coherencia con lo anterior, no se presenta como un ejemplo, sino como una singularidad dentro del conjunto del estado. 


\section{Educación de personas adultas en Andalucía (1982-)}

Voy a presentar la evolución de la EA en Andalucía considerando cuatro asuntos y momentos diferentes. i) El relámpago de esperanza que la EA trajo después de los negros años de la Dictadura, ii) los esfuerzos de los respectivos gobiernos - siempre del Partido Socialista Obrero Español - por hacer retroceder la EA y encerrarla en la escuela, lejos de las comunidades, iii) esos mismo esfuerzos se concretan en romper los lazos entre las escuelas y las comunidades donde se encuentran, y iv) la resistencia y la creatividad de las personas por mantener una EA que suponga un proceso de ilustración y liberación.

La evolución política que hemos descrito en los apartados anteriores nos debe ayudar a entender la laguna existente entre los deseos de las personas por alcanzar un nivel educativo - aunque sea elemental - y las actuaciones - o, en muchas ocasiones, la ausencia de actuaciones - por parte de los gobiernos. En el caso de Andalucía, el gobierno tiene poder legislativo en los asuntos educativos desde 1982. Desde esa fecha el mayor desafío, en relación a la EA, ha sido reducir los altos niveles de analfabetismo. De hecho, este objetivo forma parte del discurso de investidura del primer presidente electo del gobierno de Andalucía en 1983.

\section{El primer esfuerzo por los derechos}

Los documentos oficiales referidos a la EA en Andalucía comienzan explicando que en el principio había 36 profesores y 1900 estudiantes adultos: o sea, una media de 52 estudiantes por cada profesor/a. Estos documentos oficiales no intentan explicar las profundas contradicciones entre los objetivos presentados por los políticos en sus discursos y las acciones realmente desarrolladas. Desde el principio, la EA en Andalucía ha sido una primorosa herramienta de propaganda utilizada por los diversos gobiernos - repito, siempre del mismo partido - para demostrar su dedicación a la gente, siempre a la manera de un buen padre.

En cualquiera de los documentos oficiales sobre la EA en Andalucía que examinemos, es imposible encontrar la historia de este proceso - muy específico en cada lugar, ciudad, barrio, pueblo pequeño - que permitió desarrollar la EA en Andalucía. Para que tengamos una idea que lo que ocurrió, podemos usar el ejemplo de una escuela singular construida por las ciudadanas y los ciudadanos del barrio de Parque Alcosa en Sevilla. En 1981, al principio de lo que podemos definir como la era creativa y romántica (Rubenson, 2004) el barrio estaba situado dos kilómetros fuera de la ciudad. Siete personas, dos hombres y cinco mujeres, con diplomas universitarios, no solo en educación, fueron a colaborar con los vecinos y vecinas de ese barrio, que los habían animado a trabajar con las personas analfabetas. La única cosa en común de esas siete personas es que formaban parte de un movimiento de renovación pedagógica llamado Colectivo Andaluz de Pedagogía Popular.

Estos hombres y mujeres que fueron en 1981 a trabajar con las vecinas y los vecinos lo hicieron de forma voluntaria. En el segundo año de trabajo, la asociación de vecinos les pagaba el autobús desde Sevilla al barrio. Al fin, entre el tercer y el cuarto 
año de trabajar con las personas del barrio los maestros y maestras comenzaron a recibir un salario del Ayuntamiento de Sevilla.

Este ejemplo es muy usual en los inicios de las escuelas para personas adultas y de la EA en Andalucía, tanto en los pueblos pequeños como en los barrios de las grandes ciudades. De hecho, durante esos días románticos, una frase recorría todo Andalucía, no como un fantasma, sino como un destello de esperanza: En un pueblo bay dos lugares abiertos por la noche: el bar y la escuela de educación de personas adultas. Hoy, más de treinta años después, sólo el bar permanece abierto.

Este periodo estaba en su esplendor cuando el Curriculum de Educación de Adultos (sic) entro en vigor en 1985. El documento al que hacemos referencia plantea dos asuntos importantes: i) por un lado, el curriculum define la EA no sólo como un trabajo académico, sino como un trabajo socioeducativo que sólo puede tener lugar en las comunidades donde las personas viven. Esto establecía relaciones entre los procesos de desarrollo comunitario - muy importantes en ese momento en las zonas rurales de Andalucía - y el animar a las personas a asistir a las acciones educativas en una perspectiva freireana. ii) Por otro lado, este marco socioeducativo estaba basado en una clase de modelo territorial en el que las personas enseñaban sobre su territorio que se convertía en la semilla para los procesos de enseñanza/aprendizaje. El curriculum definía una metodología caracterizada por una serie de elementos tales como: i) activa en el sentido de que cada persona era el sujeto de su propio aprendizaje, ii) dialogal, sobre todo en relación a los diferentes niveles de comunicación, iii) participativa. De hecho, el curriculum animaba a las personas a acometer procesos de investigación participativa y presentaba esta como la principal metodología de trabajo. En el curriculum de 1985, la investigación participativa era la conexión entre la escuela y la vida cotidiana de las personas, conectándola con el desarrollo comunitario.

Este creativo y participativo proceso termina cuando la Ley de Educación de Adultos de Andalucía - la primera en España y hoy no existente - fue promulgada en 1990. Esta ley fue el canto de cisne de este creativo, espontaneo y romántico periodo de construcción de una EA liberadora al servicio de las personas y las comunidades.

\section{De la investigación participativa a la educación bancaria}

En 1990, como ya hemos indicado, entro en vigor la LOGSE. Esta ley es particularmente importante ya que amplió la educación obligatoria hasta los 16 años, iniciando una Educación Secundaria Obligatoria que ampliaba los horizontes de las personas. Para la EA supuso también un profundo cambio. De repente las prioridades del curriculum de 1985 cambiaron: de la alfabetización y una educación universal de base se paso a la expedición del diploma en Educación Secundaria Obligatoria.

Es bastante irónico que la LOGSE entrara en vigor al mismo tiempo que la Ley de Educación de Adultos de Andalucía. Y es irónico porque cada propuesta hecha en la ley andaluza fue eliminada cuando se comenzó a desarrollar la propia LOGSE. 1990 puede ser considera el año en que la EA como un proyecto comunitario comenzó a retroceder. 
Los efectos de potenciar la educación secundaria en detrimento de la educación elemental, y me refiere específicamente a las personas adultas, fueron algunos como los siguientes: i) el abandono de las personas analfabetas a su suerte - el curriculum de 1997 limitaba el número de años que una persona podía dedicar a llegar a ser alfabeto. En Andalucía, un/a estudiante adulto/a podía permanecer en los niveles de alfabetización dos años, después de ellos debería abandonar la escuela; ii) supuso también el final de un proyecto basado en el territorio más cercano a las personas como fuente de los aprendizajes. La educación deviene aprendizaje de materias determinadas y específicas y se recluye en la escuela. De alguna forma es la desaparición de la EA como un proyecto y campo específico de actuación.

Finalmente, en 2007 fue promulgada la Ley de Educación de Andalucía. Con esta ley se cierra, de alguna forma, el ciclo legislativo ya que esta norma abole la ley especifica sobre educación de personas adultas de 1990. La ley de 2007 ya no habla de EA sino que acuña un siempre confuso término: Educación Permanente de Adultos y se centra, siguiendo los dictados provenientes de la Comunidad Europea en el entrenamiento para ser empleables en el mundo del trabajo.

\section{Del modelo territorial al modelo escolar}

Si alguna cosa puede considerarse especialmente característica del comienzo y desarrollo temprano de la EA en Andalucía esta es, sin duda, la estrecha relación construida y mantenida entre las escuelas y los territorios donde éstas se encontraban. De hecho, en sus inicios fue denominado como un modelo territorial debido a las estrechas relaciones que se construían entre la escuela y la comunidad. Lo más importante en todo esto era como las tareas escolares, los conocimientos construidos al interior de las escuelas de personas adultas derivaban de las palabras y los temas generadores, estrechamente relacionados con el entorno y la vida cotidiana de las personas. Esta significación es fundamental porque la EA fue una especie de faro cultural, social y educativo en los barrios y en los pueblos. Cuando el poder de transformación que esta clase de educación llevaba consigo fue descubierto por los gobiernos, el esfuerzo de estos fue hacerla desaparecer, o, al menos, hacerla invisible y situarla en un lugar marginal en términos sociales y educativos.

De esta forma, se consideró y se transmitió el mensaje de que la EA solo podía ser trabajada en la escuela, protegida de los peligros del mundo exterior. El Desarrollo Comunitario, por ejemplo, se convirtió, en el curriculum de 1997 en una asignatura que debía enseñarse tres o cuatro horas a la semana. El mercado también entró en la escuela: el principal objetivo para EA es, hoy, incrementar las cualificaciones de la población para satisfacer las necesidades del mercado de trabajo.

El último paso fue el reduccionismo de la EA. En 2006, el gobierno autónomo abandono el modelo territorial a cambio de un modelo burocrático cuya primera finalidad era controlar los procesos de enseñanza/aprendizaje y estandarizar el curriculum y las actividades.

Y, finalmente, la sociedad del conocimiento apareceré. La larga y oscura sombre del ALV entra en el modelo de EA en Andalucía. Un antiguo presidente del 
gobierno autónomo - hoy pendiente de juicio en lo tribunales - señala como la EA debe adecuarse a los nuevos requerimientos de la sociedad del conocimiento y las nuevas demandas que aparecen debido al desarrollo de la economía global (Chaves, 2008).

Quizás es este el mejor epitafio para enterrar un modelo educativo basado en la lucha contra las tendencias homogeneizadoras y despersonalizadoras del mercado, y que pretendió dar una respuesta creativa a los deseos y los intereses de las personas en su vida compartida dentro de las comunidades.

\section{En las grietas del sistema hay un nuevo (re)comienzo}

Pero es muy difícil hacer invisibles los deseos y los derechos de las personas. La EA en Andalucía ha hecho un largo viaje desde los siete de Alcosa en autobús como ejemplo de muchos otros y otras siete, cuatro o dos. Por desgracia, este viaje ha dejado de mezclar la escuela y la comunidad. La escuela ha sido limitada y conducida hacia el mercado. Pero la realidad sale por la puerta y entra por la ventana y este viaje fue hecho por las personas construyendo contra las fuerzas que han intentado eliminar la EA. Ha sido un viaje lleno de experiencias que nos muestran como las conexiones entre la escuela y la comunidad han sido muy fructíferas.

Pero, fuera de los estrecho muros en los que se ha situado a la escuela puede verse una nueva esperanza que aparece: aprendices - en un auténtico enfoque freireano - que organizan el retorno de las viejas y antiguas prácticas de una EA que sea apropiada y responda a sus problemas y deseos. Es retornar a la lucha por los derechos, para que la educación siga significando decir la palabra, comprender el mundo y transformarlo. Significa también el retorno a la comunidad, a los temas que nacen de las preocupaciones de las personas en su vida cotidiana. Es el caso de las mujeres que se auto organizan para construir su propio taller de teatro, o aquellas personas que bucean en su pasado para rescatarlo del olvido y recuperar la memoria. Estos y otros ejemplos son la promesa de que la EA se mantiene construyendo en su trabajo liberador y de comprensión y transformación de la realidad.

\section{Conclusiones}

La educación de personas adultas en Andalucía comenzó como un camino para superar el histórico retraso en los niveles de analfabetismo. La principal metodología para acometer la tarea y superar el retraso fue situar a las personas y las comunidades en el centro del trabajo. En ese sentido la EA intentó trabajar en una línea educativa y comunitaria uniendo la cultura, la educación, el desarrollo comunitario, etc. Por ello, se puede considerar que, al menos hasta 1990, o quizás 1995, la EA que creció en Andalucía estaba relacionada con las principales finalidades de un EA liberadora. Lo esencial de ese período fueron las estrechas relaciones entre las personas, los y las educadoras y educadores e, incluso, los políticos - sobre todo a nivel municipal - para construir una educación orientada a edificar una sociedad más igualitaria.

Desde 1990 - sobre todo 1995 - el sistema cambió. Y este cambio, como he señalado más arriba, está conectado con los dos elementos fundamentales y característicos de las políticas y las prácticas del ALV. 
En primer lugar el retorno a la escuela, la primacía de lo escolar. Las cosas más importantes que una persona - incluida una persona adulta - puede aprender están en un libro que se enseña en la escuela - ahora en internet. El curriculum de 1997 es muy claro cuando señala, de forma muy detallada, que cosas debe aprender una persona adulta y en qué orden debe aprenderlas. El abandono de las personas analfabetas significa un cambio en las grandes finalidades de la educación: no se trata de ayudar a las personas, se trata de crear una persona con un diploma.

El segundo elemento está relacionado con los discursos gerencialistas como destacaba Lima (2000). Lo más importante es la gestión de los asuntos administrativos. Por ejemplo, en una escuela de educación de personas adultas se elaboro un proyecto muy creativo con una gran cantidad de actividades dirigidas a las vecinas y los vecinos del barrio - incluyendo gran un número de personas inmigrantes. La respuesta que recibieron de las autoridades educativas fue que las horas dedicadas a las tareas de aprendizaje y enseñanza no estaban claras. Lo más importante es alejar a la escuela de la comunidad y acercarla a las grandes empresas.

En resumen, por desgracia es posible decir que una EA creativa dedicada a ayudar a las personas a llegar a ser ciudadanos y ciudadanas ha sido encerrada en los estrechos muros de la escuela, evitando las relaciones entre las personas y con sus comunidades. Y esta tarea ha sido hecha en Andalucía en nombre del Aprendizaje a lo largo de la vida.

\section{Referências}

CHAVES, M. Educación de personas adultas en Andalucía. Trasatlántica, 2, 2008, p. 13-16.

COMUNIDAD EUROPEA. A Memorandum on Lifelong Learning. SEC (2000) 1832.

COMUNIDAD EUROPEA. Communication from The Commission to The Council, The European Parliament, The European Economic and Social Committee and The Committee of the regions. It is always a good time to learn. COM (2007) 558 final.

CROWTHER, J., JOHNSTON, R., MARTIN, I.; MERRILL, B. Defending the radical margins of university adult education. In: ANTIKAINEN, A.; HARINEN, P.; TORRES, C.A (Eds.). In from the margins (p. 53-64). Rotterdam: Sense Publisher, 2006.

EDWARDS, R. Actively seeking subjects? In: FEJES, A.; NICOLL, K. (Eds.). Foucault and Lifelong Learning. Governing the subject (p. 21-33). London: Routledge, 2008.

FLECHA, R.; LÓPEZ, F.; SACO, R. Dos siglos de Educación de Adultos. De las sociedades de Amigos del País a los modelos actuales. Barcelona: El Roure, 1988.

FREIRE, P. Pedagogía del Oprimido. Madrid: Siglo XXI, 1985.

GELPI, E. Educación Permanente. Problemas laborales y perspectivas educativas. Madrid: Popular, 1990. 2004.

Trabajo Futuro. La formación como proyecto político. Xàtiva: Edicions del CREC,

GELPI, E. El trabajo: utopía cotidiana. Xàtiva: Edicions del CREC, 2008.

GOMES, I.; LUCIO-VILLEGAS, E. Recognition of lifelong and lifewide learning within the scope of Permanent Education. A new pathway towards citizenship? In: LUCIO-VILLEGAS, E. (Ed.), Citizenship as Politics. International perspectives from Adult Education (pp. 71-90). Rotterdam: Sense Publisher, 2009. 
HILL, R. Critical Indigenism and Adult Learning and Education. In: GANDY, P.; TIESZEN S.; TAYLOR-HUNT, C.; FLOWERS, D.; SHEARED, V. (Eds.), Adult Education, the next 50 years: our future continues through an investment in Research (pp. 184-189). Sacramento, CA: Sacramento State University, 2010.

JARVIS, P. Sociología de la educación continua y de Adultos. Barcelona: E1 Roure, 1989.

LENGRAND, P. Introducción a la Educación Permanente. Barcelona: Teide/ UNESCO, 1973.

LIMA, L. C. Educação de Adultos e construção da cidadania democrática: Para una critica do Gerencialismo e da educaçao contabil. In: LIMA, L. C. (Org.). Educaçao de Adultos. Forum I. Braga: Universidade do Minho, 2000.

LUCIO-VILLEGAS, E. Introduction. In: LUCIO-VILLEGAS, E. (Ed.). Citizenship as Politics. International perspectives from Adult Education (p. xiii-xv). Rotterdam: Sense $\mathrm{Pu}-$ blisher, 2009c.

Basic skills for becoming citizen. In ZARIFIS, G. K.; GRAVANI, M. (Eds.). Challenging the 'European Area of Lifelong Learning': a critical response (p. 41-50). Dordrecht: Springer, 2004.

Adult Education in Communities. Approaches from a Participatory Perspective. Rotterdam: Sense Publishers, 2015.

NICOLL, K.; FEJES. A. Mobilizing Foucault in Studies of Lifelong Learning. In: FEJES, A.; NICOLL, K. (Eds.), Foucault and Lifelong Learning. Governing the subject (pp. 1-18). Londres: Routledge, 2008.

RUBENSON, K. Lifelong learning: A critical assessment to the political project. In: ALHEIT, P.; BECKER-SCHMIDT, R.; GITZ-JOHANSEN, T.; PLOUG, L.; SALLING OLESEN, H.; RUBENSO, K. (Eds.) Shaping an Emerging Reality. Researching Lifelong Learning. Roskilde: Roskilde University, 2004.

VIÑAO, A. Historia de un largo proceso. Cuadernos de Pedagogía, 179, 45-50, 1990.

* Professor da Universidade de Sevilla, Sevilla, Espanha.

\section{Correspondência}

Emilio Lucio-Villegas - Universidad de Sevilla - Dpto. de Teoria e História de la Educacíon y Pedagogia Social. Faculdad de Ciencias de la Educación. Calle Pirotecnia s/n. Despacho 5120, 5a Planta. CEP: 41013. Sevilla, Espanha.

E-mail: elucio@us.es

Recebido em 28 de janeiro de 2017

Aprovado em 29 de março de 2017 